\title{
Optimal Performance of Modular and Synchronized Mechatronic Systems
}

\author{
Sathyamyla Kanthabhabhajeya, Bengt Lennartson \\ Chalmers University of Technology \\ SE-41296 Gothenburg, Sweden \\ satkan@chalmers.se
}

\begin{abstract}
Optimizing the configuration and overall performance of synchronized modular systems is considered in this paper. The synchronized modules can be considered as a hybrid system, including continuous-time dynamics of local moving devices, combined with high-level discrete event sequences. The continuous-time trajectories are approximated by the Gauss pseudospectral method, resulting in a nonlinear programming (NLP) problem. The optimal configuration generates the maximal production rate subject to dynamic constraints. A complete design procedure is presented and applied to a case study of a packaging machine, where an alternative optimal configuration is achieved compared to current industrial practices.
\end{abstract}

\section{Introduction}

In manufacturing industries, it is quite common that new hardware technologies are combined with traditional design methods. By these traditional methods, the overall system performance is affected and the full advantage of new technologies is not used. Especially in package solution industries, previous generation mechanically driven systems are replaced with mechatronic devices. These mechatronic systems include different modules, but they are synchronized to a single master in the same way as traditional mechanical drive systems. The existing solutions include continuous-time dynamics of moving devices along with discrete event sequences. The modeling of mechatronic package machines with continuous and discrete behavior along with synchronization are discussed in detail in $[1,3,4,6,13]$.

Optimization is often a natural part of system design, even during early configuration phases. A mechatronic system, involving mixed continuous and discrete behavior, is generally a hybrid system [7]. Often there is a clear separation between the continuous and the discrete part, meaning that the continuous parts may first be evaluated before the higher level discrete parts are considered. Especially, this is the case for mechatronic systems where the movements of individual components are synchronized with a master profile. The optimization of such hy- brid systems is considered in this paper, where each local module of the system defines an optimal control problem, which is later combined with shared variables for synchronization. Along with this, high-level discrete sequences of operations are also defined representing the transition between discrete states.

Optimal control problems are often solved numerically by collocation methods [10]. The original continuous optimization problem is then only considered at discrete collocation points, and the approximate optimization formulation is transformed to a Nonlinear Programming (NLP) problem, [11,12], which can be solved by solvers such as bonmin [5]. One common approximation, applied in this paper, is the Gaussian pseudospectral method (GPM) [16], where the continuous trajectory is approximated by a Lagrange polynomial and the trajectory is only optimized at discrete time instances.

An application of the GPM is presented in this paper for optimization of a packaging machine during its early stages of design configuration. The resulting effect of the optimal solutions are verified for different modules. The bridge between discrete sequences of operations and the continuous dynamics is also modeled.

The main contributions of this paper are as follows: Early configuration design of a mechatronic system is formulated as optimization of a modular hybrid system. A clear decoupling of the continuous and the discrete behavior makes it possible to solve the problem in two steps, including continuous trajectory optimization followed by a high level configuration and operation sequence optimization.

A filling machine (TR/28) from Tetra Pak, package solution industry is used as a case study in this paper. Optimal control problems are defined, including continuous and discrete dynamic constraints, for the index conveyor and the filling module (Lift and Pump) of the filling machine. As a result of this optimization, an interesting alternative optimal configuration is obtained, compared to industrial practice.

This paper is organized as follows. In Section 2, the continuous optimization problem, including its approximation, is presented. Section 3 describes the modeling of discrete event sequences. This is followed by the case study in Section 4 and a discussion and conclusions in 
Section 5.

\section{Continuous Optimal Control}

In this section the continuous optimal control problem is formulated, followed by an approximation based on the Gauss pseudospectral method.

\subsection{Optimal Control}

A continuous optimal control problem is modeled to determine a control input that allows the system to follow a state variable or trajectory and at the same time, minimize or maximize the chosen performance criteria [15]. A standard performance index is chosen in the optimal control problem formulation.

$$
J=\Phi\left(\mathbf{x}\left(t_{f}\right), t_{f}\right)+\int_{t_{0}}^{t_{f}} g(\mathbf{x}(t), \mathbf{u}(t), t) d t
$$

where $\Phi: \mathbb{R}^{n} \times \mathbb{R} \rightarrow \mathbb{R}$ is the terminal cost and $g: \mathbb{R}^{n} \times$ $\mathbb{R}^{m} \times \mathbb{R} \rightarrow \mathbb{R}$ is the integral cost. In this cost function, the state variables are subject to first-order dynamic constraints.

$$
\dot{\mathbf{x}}=\mathbf{f}(\mathbf{x}(t), \mathbf{u}(t), t)
$$

where $f: \mathbb{R}^{n} \times \mathbb{R}^{m} \times \mathbb{R} \rightarrow \mathbb{R}^{n}$. The boundary conditions and algebraic path constraints that restricts the state variables are described by,

$$
\begin{aligned}
\psi\left(\mathbf{x}\left(t_{0}\right), t_{0}, \mathbf{x}\left(t_{f}\right), t_{f}\right) & =0 \\
b(\mathbf{x}(t), \mathbf{u}(t), t) & \leq 0
\end{aligned}
$$

with $\psi: \mathbb{R}^{n} \times \mathbb{R} \times \mathbb{R}^{n} \times \mathbb{R} \rightarrow \mathbb{R}^{q}$ and $b: \mathbb{R}^{n} \times \mathbb{R}^{m} \times \mathbb{R} \rightarrow \mathbb{R}^{r}$. The inequality constraints relating to states and control are included in the path constraints $b \leq 0$.

\subsection{Continuous-time Approximation}

The nonlinear optimal control problem (1) - (3) is solved numerically by GPM, where Lagrange polynomials are used to approximate the states. The states have coefficients of initial state and $N$ collocation points. The set of collocation points used are either Legendre-Gauss(LG), Legendre-Gauss-Radau or Legendre-Gauss-Lobatto, and these are obtained from the roots of Legendre polynomial [9]. The set of points are defined in the domain $[-1,1]$. In this paper, $\mathrm{LG}$ points are used that include neither of the endpoints.

The polynomial $X(t)$, the state vector approximation is of degree $N$ and hence requires $N+1$ points to define its derivative. Let $L_{k}(t), k=0, \ldots, N$ be a basis for Lagrangian polynomial on the interval $[-1,1][8]$. Then,

$$
L_{k}(t)=\prod_{\substack{i=0 \\ i \neq k}}^{N} \frac{t-t_{i}}{t_{k}-t_{i}}, \quad k=0, \ldots, N
$$

from which it is seen that,

$$
L_{k}\left(t_{i}\right)= \begin{cases}1 & \text { if } k=i \\ 0, & \text { if } k \neq i\end{cases}
$$

The state vector $x(t)$ is approximated by $X(t)$ as,

$$
\mathbf{x}(t) \approx \mathbf{X}(t)=\sum_{k=0}^{N} \mathbf{x}\left(t_{k}\right) \cdot L_{k}(t)
$$

As mentioned earlier, the Lagrange polynomial is in the interval $[-1,1]$ and the interpolation points used are -1 and the $N$ Gaussian quadrature points. Differentiating (6) results in the following expression for the approximated state derivatives $\dot{X}\left(t_{i}\right)$,

$$
\dot{\mathbf{x}}\left(t_{i}\right) \approx \dot{\mathbf{X}}\left(t_{i}\right)=\mathbf{x}\left(t_{0}\right) \cdot \bar{D}_{i}+\sum_{k=1}^{N} \mathbf{x}\left(t_{k}\right) \cdot D_{i k}
$$

where, $\bar{D}_{i}=\dot{L}_{0}\left(t_{i}\right)$ and $D_{i k}=\dot{L}_{k}\left(t_{i}\right)$. The optimal control problem as defined in (1)-(3) is transformed to an NLP as follows. The performance index, (1) is approximated using Gauss quadrature such that,

$$
J=\Phi\left(\mathbf{X}\left(t_{f}\right), t_{f}\right)+\frac{t_{f}-t_{0}}{2} \sum_{k=1}^{N} w_{k} \cdot g\left(\mathbf{X}\left(t_{k}\right), \mathbf{U}\left(t_{k}\right), t_{k}\right)
$$

where $U\left(t_{k}\right)$ is an $N-1$ degree approximation of the controls $u(t)$ collocated at the Gaussian points. $w_{k}$ are the Gaussian weights; $\left(t_{f}-t_{0}\right) / 2$ comes from transforming the problem from $t \in[-1,1]$ into $t \in\left[t_{0}, t_{f}\right]$. The first order derivative of the state variables are approximated as,

$$
\begin{array}{r}
\dot{\mathbf{X}}\left(t_{i}\right)=\frac{2}{\left(t_{f}-t_{0}\right)} \mathbf{X}\left(t_{0}\right) \cdot \bar{D}_{i}+\frac{2}{\left(t_{f}-t_{0}\right)} \sum_{k=1}^{N} \mathbf{X}\left(t_{k}\right) \cdot D_{i k}= \\
\mathbf{f}\left(\mathbf{X}\left(t_{k}\right), \mathbf{U}\left(t_{k}\right), t_{k}\right), i=1, . ., N
\end{array}
$$

The boundary and path constraints from (3) are forced to be,

$$
\begin{aligned}
\psi\left(\mathbf{X}\left(t_{0}\right), t_{0}, \mathbf{X}\left(t_{f}\right), t_{f}\right) & =0 \\
b\left(\mathbf{X}\left(t_{k}\right), \mathbf{U}\left(t_{k}\right), t_{k}\right) & \leq 0, \quad k=1, \ldots, N
\end{aligned}
$$

The terminal states of the optimal control problem are followed by quadrature approximation as,

$$
\mathbf{X}\left(t_{f}\right)=\mathbf{X}\left(t_{0}\right)+\frac{\left(t_{f}-t_{0}\right)}{2} \sum_{k=1}^{N} w_{k} \cdot \mathbf{f}\left(\mathbf{X}\left(t_{k}\right), \mathbf{U}\left(t_{k}\right), t_{k}\right)
$$

The decision variables for the optimal control problem are the states $\mathbf{X}\left(t_{k}\right)$, the controls $\mathbf{U}\left(t_{k}\right)$, the initial time $t_{0}$ and the final time $t_{f}$.

\section{Discrete Event Sequences}

As described in Section 1, often mechatronic systems are considered as hybrid systems, which also establishes a 
clear distinction between continuous and discrete behaviors. Although these systems exhibit modular structures, they are later synchronized to a single master. The continuous dynamics of the system are synchronized between the modules. Hence, the discrete actions follow the time cycle of the synchronized master of the system. As described in Section 2.2, the continuous dynamics of each module is modeled with Lagrangian polynomials. The discrete event sequences of such a mechatronic system are naturally represented based on sequences of operations (SOPs) [14]. An Operation is formally defined by a three state Extended Finite Automaton (EFA), where an EFA is a generalization of an automaton including variables, guards and actions [14].

Each operation is self-contained, i.e. it holds necessary information to determine its own state and also, together with other operations, determine the relation between each other. The relation between different operations can be sequence, parallel, alternative or arbitrary order. The pre- and post-conditions of an operation, as shown in Figure 1 include guards and actions that determine the relations between operations. A sequence where operation $\mathrm{O}_{1}$ is followed by operation $\mathrm{O}_{2}$ means for instance that the pre-condition to execute $\mathrm{O}_{2}$ is that operation $O_{1}$ must be completed, that is being in location $O_{1}^{f}$. This means that $C_{2}^{\uparrow}=O_{1}^{f}$.
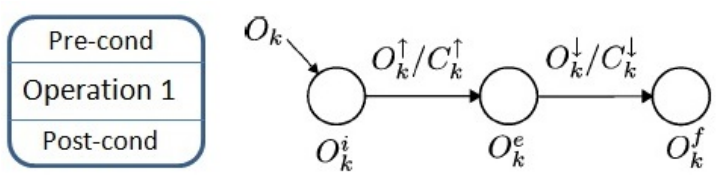

Figure 1: Three state operation model

The mechatronic system that is considered in this paper has motions that are executed simultaneously with other operations. These parallel operations are also modeled as a SOP, with a principle behavior illustrated in Figure 2. The operations, $\mathrm{O}_{7}$ and $\mathrm{O}_{8}$ are running in parallel, which means that operation $\mathrm{O}_{9}$ can be executed only when both operations $O_{7} \& O_{8}$ are in locations $O_{7}^{f} \& O_{8}^{f}$. The decoupled continuous and discrete behaviors are achieved by establishing a bridge between the two dynamics of the system. The SOPs include additional constraints from continuous dynamics, which are modeled as post-conditions on operations as shown in Figure 2. The execTime ${ }_{k}$ is a clock variable that starts at zero when an operation is initialized and the minimal operation time for a specific operation is given by $t_{f k}$. The variable $t_{f k}$ is obtained from the optimal solution of the continuous dynamics for operation $O_{k}$. Details of the SOP language and the application tool for this language are referred to $[2,14]$.

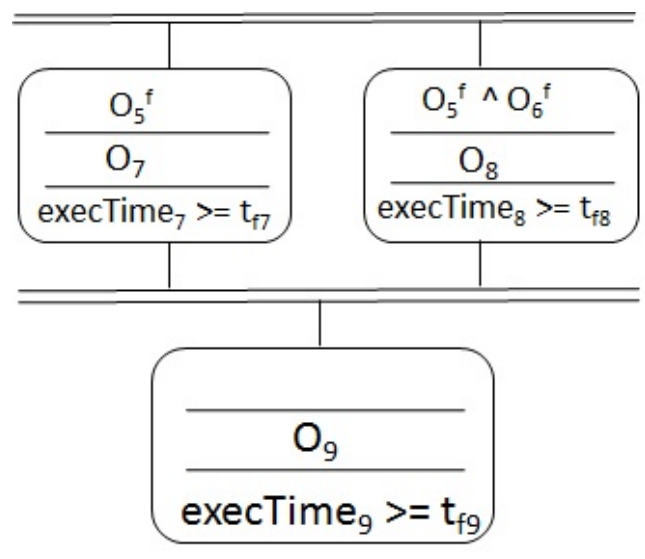

Figure 2: Examples of operations with post-conditions related to the operation time of continuous dynamics optimization

\section{Optimization of a Packaging Machine}

\subsection{Machine Description}

In this paper, a filling machine TR/28, of Tetra Pak, a packaging solution industry, is studied. This filling machine consists of five different modules, where a module is one that converts an input product to another by performing a set of operations. Feeder, bottom sealing, sterilizing, filling and top sealing are the five main modules of the TR/28 filling machine. An indexing conveyor runs through most of the modules in the machine, carrying the product. The conveyor moves in every step, i.e, it moves a certain distance and stops, for the modules to execute their sequences of operations.

This paper focuses on the index conveyor and the filling module of the entire machine. The movement of the conveyor along with cartons is tracked with its motion profile, and the constraints that control the behavior and performance are also analyzed. The lift and pump are the two main moving parts in the filling module. Once the conveyor stops, the lift moves up and down with the carton. The pump, which being connected to the tank, fills the liquid to the carton, while the lift is moving down. The conveyor starts to move again with the filled carton and the cycle repeats. The principle position curves of motion profiles of the index conveyor, lift and pump are shown in Figure 3.

The unique behavior of packaging machines is the synchronous behavior between the modules of the machine. As noticed in Figure 3, the machine cycle time is the same for conveyor, lift, pump and other movable parts of the machine. The operations are synchronized with respect to time and the cycle repeats while the machine is running. Note that in industry the modules and conveyors are synchronized with respect to angular position (in degrees) but for simplicity it is considered as time in this paper.

Apart from machine cycle time of the motion profiles, batch size of the machine also restricts the performance of 


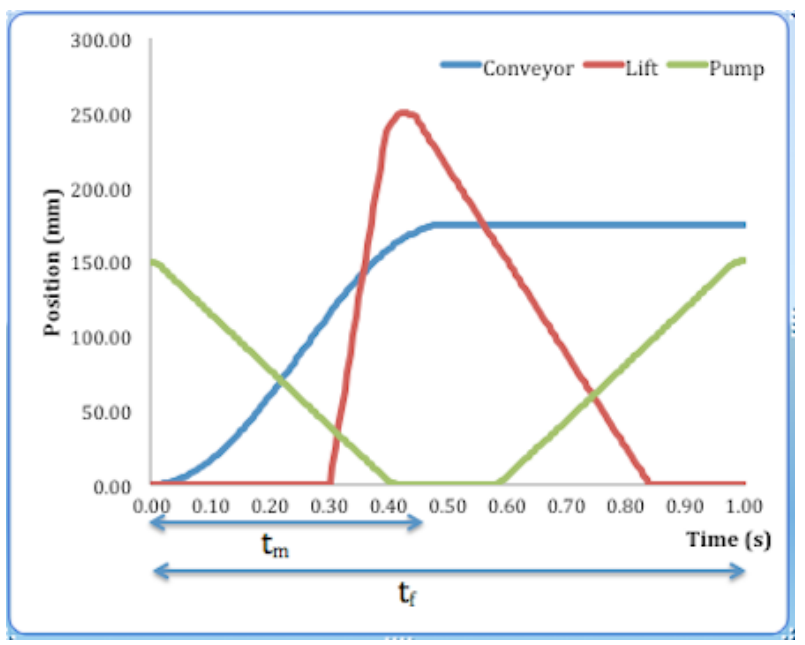

Figure 3: Principle motion profiles: position of index conveyor, lift position and pump position

the same. The number of cartons that are processed at a time defines the batch size of the machine. At present, the batch size of TR/28 is 2 . This integer variable calculates the distance to be moved by the conveyor for each step. The filling module has two lifts and two pumps that fill two empty cartons at the same time. The constraints such as space, cost, energy obtained from increasing the batch size and the resources (lift \& pump) are neglected in this study, i.e, if batch size is $n$, it is assumed that the machine will have $n$ lifts and $n$ pumps.

Another unique constraint is included to avoid slosh of the filled liquid from the carton during the index conveyor movement. In order to refrain from spilling/splashing of the liquid, Tetra Pak introduced an acceleration profile for the index conveyor. The acceleration profile is a ramp superposed by a two period sinus function with defined amplitude and frequency, as illustrated in Figure 4. The amplitude and frequency of the sinus function is proportional to indexDistance, indexTime and natural frequency of the wave. This profile is formulated based on experience and detailed flow optimization.

\subsection{Applying the Method}

The filling module and index conveyor are optimized to maximize the production rate of the entire machine. The general optimal control problem model is defined as,

$$
\left[\begin{array}{c}
\dot{x_{1}} \\
\dot{x_{2}}
\end{array}\right]=\left[\begin{array}{ll}
0 & 1 \\
0 & 0
\end{array}\right]\left[\begin{array}{l}
x_{1} \\
x_{2}
\end{array}\right]+\left[\begin{array}{l}
0 \\
1
\end{array}\right] u
$$

where the state variables $x_{1}$ and $x_{2}$ represents position and velocity respectively. The control input $u$ is the acceleration of the system, which is determined by the optimization procedure. The performance index to be minimized is,

$$
J=- \text { prodRate }
$$

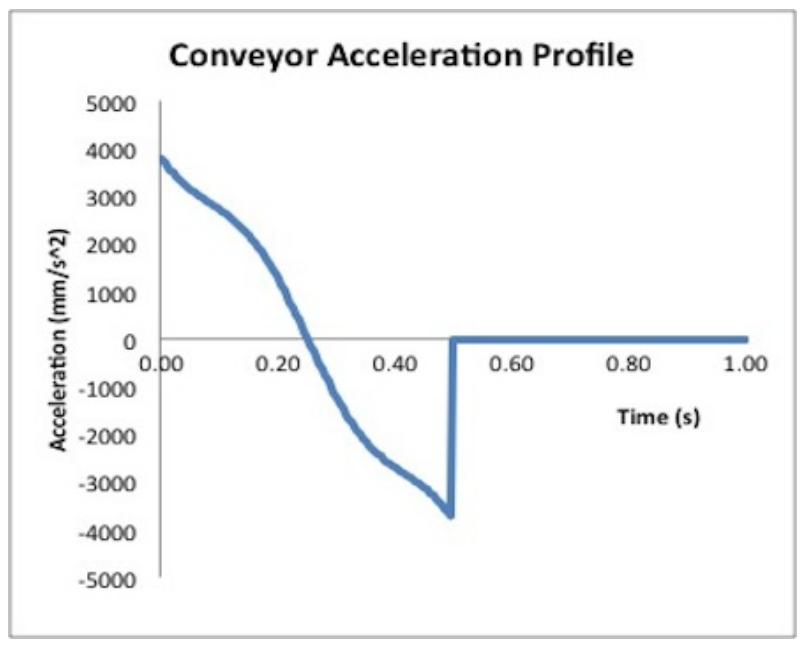

Figure 4: Principle acceleration profile of index conveyor

where prodRate $=\phi\left(t_{f}, b, x(t)\right)$ is the production rate, $t_{f}$ denoting the machine cycle time and $b$ is an integer variable representing the batch size, which varies from 1 to 10. The initial and final values of states are fed in as input data. Note, in all figures the units are in $\mathrm{mm}$.

\section{Index Conveyor}

The optimization model of the index conveyor has an objective function similar to (13). As shown in Figure 3, the index conveyor moves to a certain position at a particular time instance. In this case study, the moving time of the conveyor is considered to be a variable within the limits $0.15 s \leq t_{m} \leq 0.5 \mathrm{~s}$. The distance the conveyor travels depends on $b$ (batch size), and this adds in a constraint as

$$
x_{1}\left(t_{m}\right)=b \times \text { Width_of_Carton }
$$

In order to avoid the cartons being bounced away from the conveyor, an acceleration constraint is added to the model.

$$
|u(t)| \leq 6 \mathrm{~m} / \mathrm{s}^{2}
$$

Two different optimization models are defined for the index conveyor; one without imposing the acceleration profile and the other following the defined acceleration profile from Tetra Pak. The former model, uses Lagrange polynomial method to include dynamic constraints and optimize the prodRate. The latter defines the acceleration profile as a constraint to take as a pattern, which in turn obtain the velocity and position profiles of the index conveyor. In both models, the moving time $t_{m}$ and batch size $b$ are varied. The obtained optimal solutions of the TR/28 machine for both the index cnveyor models along with the filling module models are analyzed in Section 5.

\section{Filling Module}

The filling module (Lift \& Pump) is modeled in accordance to the four sections which includes, Lift moving up 
$\&$ down and Pump with fill \& return stroke. Again from Figure 3, it is noted that filling of the carton is executed when the conveyor is stopped and the stop time of conveyor can be denoted as $t_{s}=t_{f}-t_{m}$. From the assumption of the resources in Section 4.1, the change of batch size $b$ will not affect the production rate from the filling module. The motion profile of Lift, which moves up and down with the carton, is modeled as two optimal control problems. The same method is used for the Pump profile as well.

The profile of the Lift while moving up, includes a time span of $t_{u p}$ which varies from $0.1 s$ to $0.5 s$ with start time as the final instance of $t_{m}$. The respective end point values of the states are fed as input parameters. Similar to the conveyor, acceleration constraint is added for the Lift moving up as,

$$
|u(t)| \leq 10 m / s^{2}
$$

As the liquid is filled in the carton, while the lift is moving down, it is necessary to avoid foam being formed by controlling the flow of the liquid. The lift moving down profile with time span of $t_{\text {down }}$ is fixed to be $0.39 \mathrm{~s}$. This time span $t_{\text {down }}$ is calculated to be the shortest span, through many tests \& simulations by Tetra Pak. The initial time of the lift when it starts to move down is defined to be final instance of $t_{u p}$, and a constraint on acceleration is also included. To avoid the carton lifting from the filling module when it moves down, the negative acceleration cannot be higher than the gravity acceleration. The positive acceleration towards the end of the profile also needs to be less than $10 \mathrm{~m} / \mathrm{s}^{2}$, so to avoid slosh of liquid from the carton.

In the same way, the profiles of the Pump for fill stroke and return stroke are modeled as two optimal control problems. Additional constraints to maintain the pressure for the return stroke of the pump is included in the model. The fill stroke and the lift moving down occur simultaneously, hence with a same time span of $0.39 \mathrm{~s}$.

\section{Optimization Result}

The combined optimization model by including index conveyor, lift and pump, introduces additional constraints by defining the dependent variables. The machine cycle time $t_{f}$ is determined from the feasibility of all three dynamic parts' performance. The position, velocity and acceleration profiles of parts are described in separate subfigures for clarity. The optimal solutions, without superimposing an acceleration profile to the conveyor (cf. 4) are as shown in Figures 5a, 5c, 5e, while the results following a defined acceleration profile are shown in Figures $5 \mathrm{~b}, 5 \mathrm{~d}, 5 \mathrm{f}$. The synchronization between the modules is modeled with shared variables, cf. 4 and Figure 3.

$$
\begin{array}{cr}
t_{\text {up }, k}=t_{m, k} & k=0 \\
t_{\text {fill }, k}=t_{\text {down }, k} & k=0 . . N \\
t_{\text {down }, k}=t_{f, k} & k=N
\end{array}
$$

where $t_{u p}$ is the time span for the lift moving up; $t_{m}$, the moving time of conveyor. Similarly, $t_{\text {fill }}$, the time span for pump fill stroke and $t_{\text {down }}$ is the time span for the lift moving down.

As noticed in the resulting curves of Figure 5, the optimal solution of the model without superimposed acceleration profile yields a prodRate of 24164 cartons/hr, with a $b=4$ and $t_{f}=1.192 \mathrm{~s}$. A pre-defined acceleration profile is added as a constraint to the index conveyor model, in order to avoid the slosh of liquid from the package. This additional constraint in the model results in an optimal solution of prodRate to be 17993 cartons/hr with $b=3$ and $t_{f}=1.201 \mathrm{~s}$. The acceleration profile of index conveyor in Figure 5f, is the required trajectory to be followed for a conveyor having an indexDistance $=262.5$, indexTime $\left(t_{m}\right)=0.49263 \mathrm{~s}$ and natural frequency $=19.1 \mathrm{rad} / \mathrm{s}$.

\section{Discussion and Conclusion}

This paper presents an optimization model of a mechatronic system during the early configuration phase. Modularity of this system is used in the optimization of continuous dynamic models including adjusting the individual module criteria to obtain a feasible solution. The clear decoupling between continuous dynamics and discrete sequences make it possibile to initially optimize the continuous profiles of the system and later the discrete events following the sequences of operations. The case study of Tetra Pak TR/28 Filling Machine is based on continuous dynamics including position, velocity and acceleration of the system and the optimal results are analyzed.

As can be noticed from the optimal solutions, though the added constraint on acceleration profile reduces the performance of the machine, it is essential to avoid slosh. The slosh of a liquid needs to be refrained only after carton being filled and before the top is sealed. Due to the synchronization of the Tetra Pak machines, the index conveyor follows the same motion profiles, for the entire process. In order to reduce the constraint forced by the defined acceleration, the modules need to be de-coupled and the movement of individual cartons has to be controlled by special conveyors driven by magnetic drive systems. The magnetic conveyors allow the packages to cluster and re-cluster into different batch sizes with the help of its individually movable holders, during the process. The optimal solution for the design configuration with magnetic conveyors will be studied in the future.

It is also feasible to include net torque of the system as a constraint in the optimization model. The optimization model including the torque constraint is as an extension of this work. In addition to this, research on optimization of discrete event sequences by avoiding the strict synchronization between individual modules and movement between them will open up more flexible and optimal solution. 


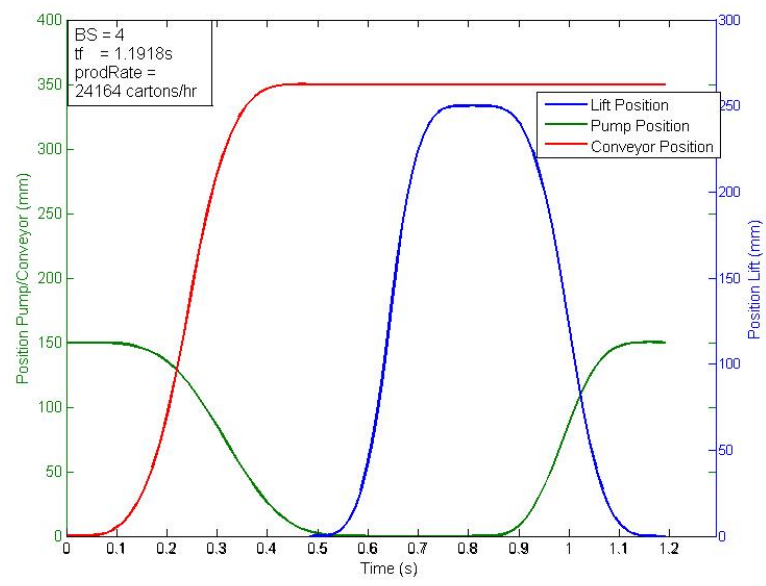

(a) Position profiles without defined acceleration

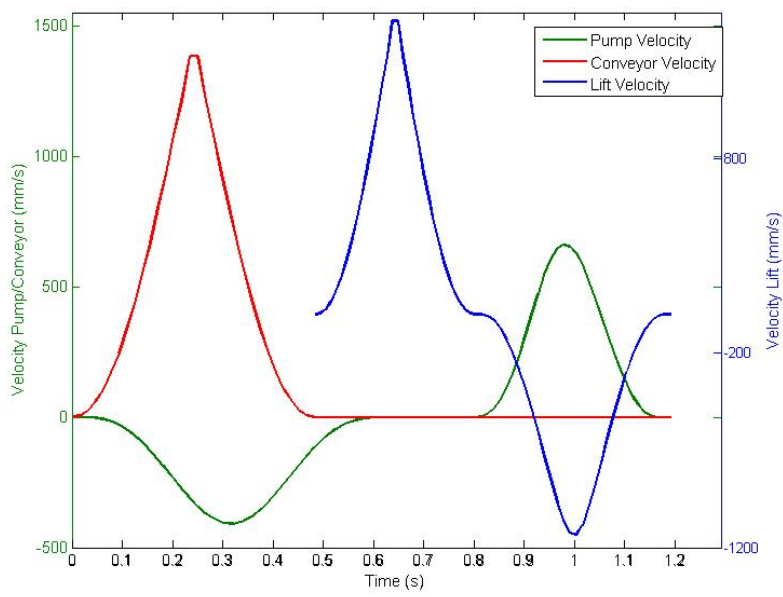

(c) Velocity profiles without defined acceleration

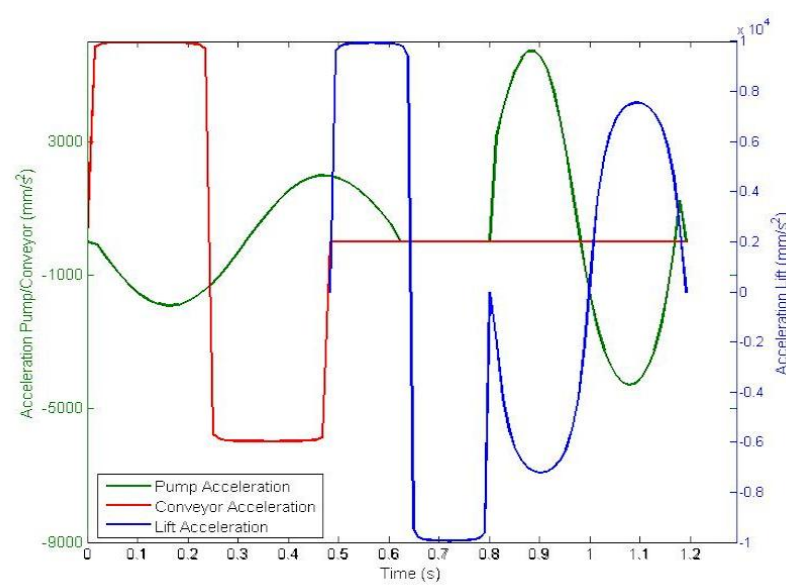

(e) Without defined acceleration profiles for index conveyor

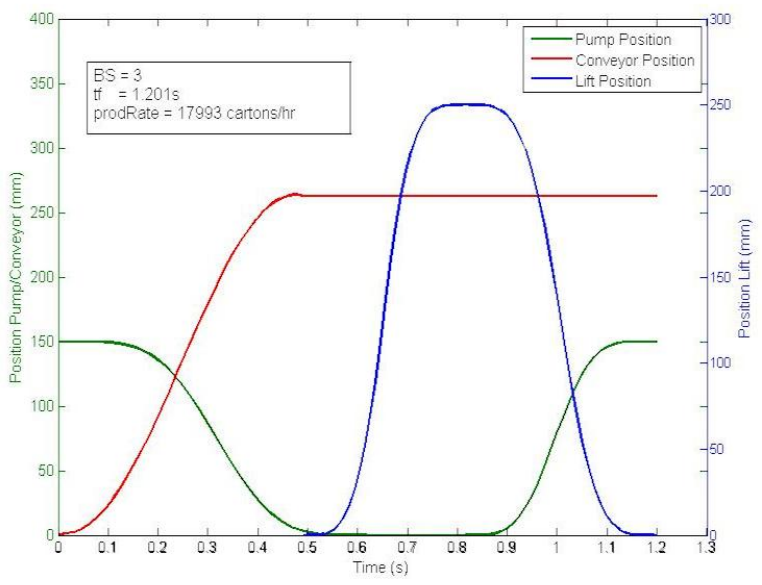

(b) Position profiles with defined acceleration

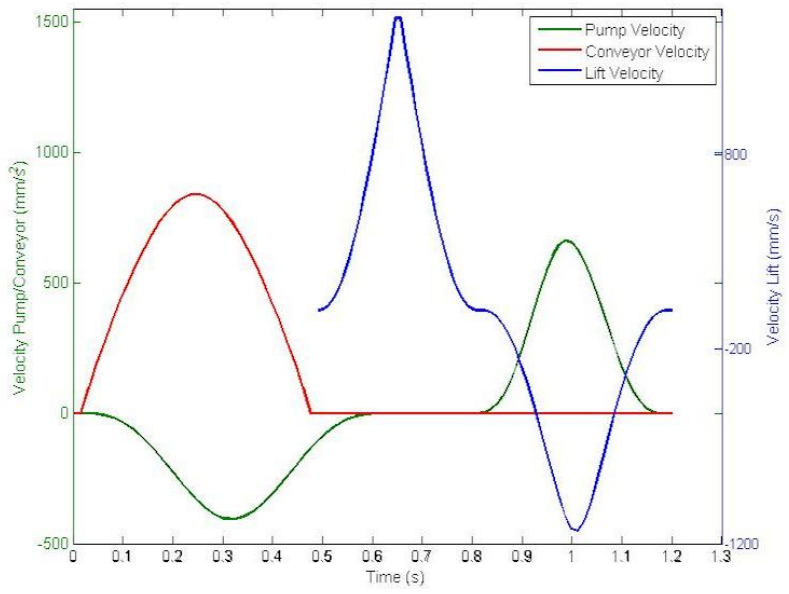

(d) Velocity profiles with defined acceleration

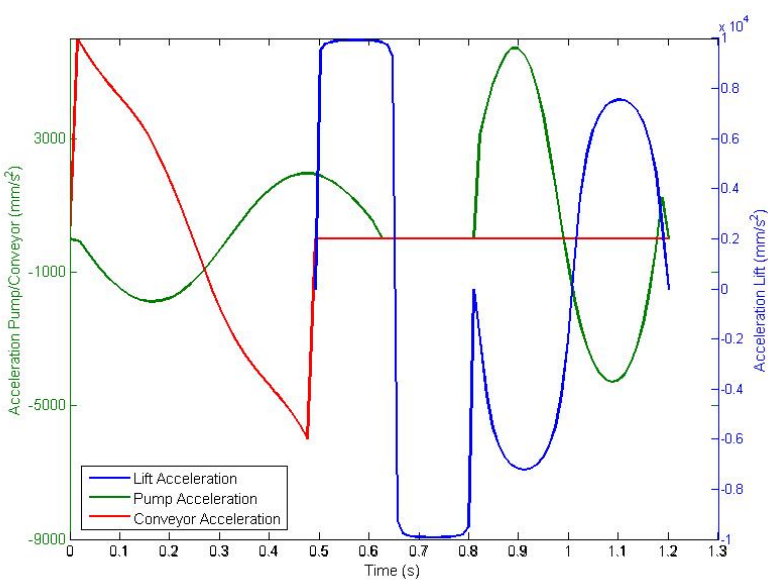

(f) Defined acceleration profile for index conveyor

Figure 5: Optimized profiles of conveyor and filling module without and with defined acceleration profile in conveyor 


\section{Acknowledgment}

This work was carried out in collaboration with and support from Tetra Pak as well as the Wingquist Laboratory VINN Excellence Center within the Area of Advance, Production at Chalmers, supported by the Swedish Governmental Agency for Innovation Systems (VINNOVA). The support is gratefully acknowledged.

\section{References}

[1] L. Bassi, C. Secchi, and M. Bonfe. A sysml-based methodology for manufacturing machinery modeling and design. IEEE/ASME Trans. Mechatronics, 16:1049-1062, 2011.

[2] K. Bengtsson. Flexible Design of Operation Behavior using Modeling and Visualization. $\mathrm{PhD}$ thesis, Chalmers University of Technology, Sweden, 2012.

[3] M. Bonfe, C. Fantuzzi, R. Borsari, and D. Borghi. Objectoriented modeling of complex mechatronic components for the manufacturing industry. IEEE/ASME Trans. Mechatronics, 12:696-702, 2007.

[4] M. Bonfe, C. Fantuzzi, and C. Secchi. Design patterns for model-based automation software design and implementation. Control Engineering Practice, 21:1608-1619, 2013.

[5] Bonmin. Bonmin wiki. http://www.ampl.com/, 2014.

[6] D. Borghi, R. Borsari, M. Poppi, E. Sacchetti, and L. Bassi. Design and modeling of a mechatronic packaging machine. Proceedings of IEEE International Symposium on Computer Aided Control System Design, pages 369-374, 1999.

[7] M. Branicky. Introduction to Hybrid Systems. Birkhuser Boston, 2005.

[8] P. Davis. Interpolation and approximation. Dover publications, 1975.

[9] G. Elnagar, M. A. Kazemi, and M. Razzaghi. The pseudospectral legendre method for discretizing optimal control problems. IEEE Transaction on Automatic Control, 40:1793-1796, 1995.

[10] D. Garg. Advances in global pseudospectral methods for optimal control. PhD thesis, University of Florida, USA, 2011.

[11] G. S. Hu, C. J. Ong, and C. L. Teo. Direct collocation and nonlinear programming for optimal control problem using an enhanced transcribing scheme. Proceedings of IEEE International Symposium on Computer Aided Control System Design, pages 369-374, 1999.

[12] C. Jansch and M. Paus. Aircraft trajectory optimization with direct collocation using movable gridpoints. Proceedings of IEEE American Control Conference, pages 262267, 1990.

[13] S. Kanthabhabhajeya, P. Falkman, and B. Lennartson. System modeling specification in sysml and sequence planner language - comparison study. Proceedings of 14th IFAC Symposium on Information Control Problems in Manufacturing Information Control Problems in Manufacturing, 2012.

[14] B. Lennartson, K. Bengtsson, C. Yuan, K. Andresson, M. Fabian, P. Falkman, and K. Akesson. Sequence planning for integrated product, process and automation design. IEEE Transactions on Automation Science and Engineering, 7:791-802, 2010.
[15] D. S. Naidu. Optimal Control Systems. CRC Press, LLC., Chennai, India, spl. indian edition, 2003.

[16] O. Wigstrom and B. Lennartson. An integrated cp/or method for optimal control of modular hybrid systems. Accepted for IEEE International Workshop on Discrete Event Systems, 2014. 